\title{
Méthodes de surveillance et de gestion des risques des médicaments pour la future mère, l'embryon, le fotus et le nouveau-né
}

\author{
Evelyne Jacqz-Aigrain, ${ }^{1}$ Véronique Lamarque ${ }^{2}$ et les participants à la table ronde $n^{\circ} 5$ de Giens XXI ${ }^{\star}$ \\ 1 Pharmacologie Pédiatrique et Pharmacogénétique, Hôpital Robert Debré, Paris, France \\ 2 Laboratoires Pfizer, Paris, France
}

\begin{abstract}
Mots clés :
gestion des risques; médicaments ; grossesse ; pharmacovigilance ; réseau

Résumé - Les données évaluant le risque d'un nouveau produit sur le déroulement normal de la grossesse et le développement de l'embryon, du fœetus et du nouveau-né sont le plus souvent manquantes lors de la mise sur le marché d'un nouveau produit. Dans une telle situation, un plan de gestion de risque doit être préparé par l'industriel et validé par les autorités de santé. Ce plan doit tenir compte des données acquises sur le médicament et de son utilisation thérapeutique potentielle par des femmes en âge de procréer. L'obtention de données humaines post-AMM (autorisation de mise sur le marché) doit s'appuyer sur les nombreux acteurs tant privés que publiques impliqués dans le recueil des données et leur évaluation. Leur organisation en réseau devrait permettre de les fédérer et d'optimiser leur action en harmonisant les données recueillies et leur suivi. Ceci permettrait de générer ou répondre rapidement à une alerte, de réaliser des études collaboratives en pharmacologie et pharmacovigilance.
\end{abstract}

\section{Introduction}

Les médicaments pris en cours de grossesse peuvent potentiellement affecter le déroulement normal de la grossesse et retentir sur le développement embryonnaire et fœtal. Les études cliniques et en particulier les essais thérapeutiques sont difficiles à conduire chez la femme enceinte, et les contraintes de l'évaluation du rapport bénéfices/risques d'un médicament sont différentes de celles qui caractérisent les essais cliniques, conduits en dehors de la grossesse. Les plans de gestion du risque, nouveau dispositif réglementaire, doivent être adaptés à cette situation particulière.

\subsection{Situations cliniques en pratique}

L'évaluation du risque des médicaments pour la femme enceinte, l'embryon, le fœtus, le nouveau-né et l'enfant peut correspondre à deux situations :

- il peut s'agir d'une évaluation individuelle du risque, mettant en présence une femme enceinte et son médecin, ce dernier devant évaluer le risque d'une prise médicamenteuse qui a déjà

^ Pour la liste des participants, voir en fin d'article. eu lieu, ou le risque du médicament qu'il souhaite prescrire. Cette situation pose le difficile problème pour le praticien, de l'accès à une information de qualité et de l'extrapolation des données populationnelles à une situation individuelle;

- il peut aussi s'agir de l'évaluation et de la gestion du risque des médicaments à l'échelon d'une population, qui pose éventuellement une question de santé publique et dans ce cas la problématique est bien différente.

Seul ce dernier aspect a été discuté au cours de la table ronde.

\subsection{Consommation des médicaments chez la femme enceinte : données épidémiologiques}

De nombreux travaux ${ }^{[1-3]}$ ont souligné une consommation médicamenteuse importante chez la femme enceinte. Depuis 2000, les études publiées en France ont montré des taux de prescription allant de 93,5\% dans la Loire avec une moyenne de 10,9 médicaments par femme dont 3,5 au cours du premier trimestre, à $99 \%$ en Haute-Garonne, avec une moyenne de 13,6 médicaments par femme, dont 5,2 au cours du premier trimestre. 
Ce taux global de prescription est significativement plus élevé que celui rapporté dans d'autres pays : 80 à $86 \%$ aux Etats-Unis, $85 \%$ en Allemagne, $68 \%$ au Royaume Uni ou encore $42 \%$ en Suède. ${ }^{[4-7]}$ Ces différences sont probablement liées à des différences de prise en charge de la femme enceinte dans ces divers pays. A titre d'exemple, l'utilisation importante des veinotoniques est très particulière à la France.

\section{Etat des lieux / Acquis des tables rondes précédentes}

Trois tables rondes précédentes ont permis de discuter certains aspects essentiels des problèmes posés par l'exposition à un médicament en cours de grossesse.

L'apport des études de toxicologie de la reproduction à l'évaluation des risques en cours de grossesse a été discuté en 1998, la conférence internationale sur l'harmonisation ayant servi de base au travail réalisé. Les conclusions de la table ronde ont porté sur plusieurs points : la nécessité d'harmoniser la réalisation et l'interprétation de ces études, leur valeur prédictive très variable, importante pour les études de tératogenèse, plus limitée pour les études de toxicité fœtale ou post-natale. ${ }^{[8]}$

En 1997 puis en 2002 ${ }^{[9,10]}$ ont été discutées les méthodologies d'évaluation du risque médicamenteux chez la femme enceinte et les modalités de gestion de ce risque. Il sera fait ici référence aux conclusions de cette table ronde, en particulier pour ce qui concerne les données pré-cliniques, cliniques et leur exploitation.

Ces tables rondes permettent une première approche de la gestion du risque chez la femme enceinte. Ainsi, les études de tératologie expérimentale ayant une bonne valeur prédictive d'un risque humain, un médicament tératogène ne sera mis ou maintenu sur le marché que dans des cas exceptionnels et encadrés d'un plan de gestion de risque renforcé. L'absence d'effet tératogène lors des études pré cliniques, est un élément très rassurant, puisqu'il n'existe pas à l'heure actuelle, de médicament évalué comme non tératogène par les études animales bien conduites ${ }^{[11]}$ pour lequel ait été démontré un effet tératogène dans l'espèce humaine.

\section{Les nouvelles réglementations européennes relatives au médicament après la mise sur le marché}

Le cadre réglementaire régissant le suivi post-AMM des médicaments s'est complété ces dernières années. En novembre
2004, les recommandations générales ICH E2E, Pharmacovigilance planning, CPMP/ICH/5716/03 ${ }^{[11]}$ étaient adoptées en novembre 2004, définissant les règles de planification des activités de pharmacovigilance dans la période suivant la mise sur le marché d'un médicament. L'identification des risques importants, avérés durant le développement clinique, ou potentiels ainsi que les données manquantes en particulier dans des sous-populations non étudiées, permettent de proposer des actions spécifiques selon ces différents risques. Le cas de la femme enceinte est au premier plan, car cette population est généralement exclue des essais cliniques en période pré-AMM, et relèvent par précaution d'une contre-indication dans le résumé des caractéristiques des produits.

La mise en place de la nouvelle législation pharmaceutique en novembre $2005^{[12]}$ a complété ces recommandations par le concept de plan de gestion de risque où l'on retrouve en plus des propositions d'actions et ou d'activités une évaluation des besoins de réduction des risques ainsi que la préparation du plan les décrivant, avec si nécessaire un plan de communication, notamment des lettres aux professionnels de santé.

Le plan de minimisation des risques peut comporter différents types d'action : guides de prescription, brochures patient, programme de formation, modification des conditions de prescription, de dispensation... Ces mesures doivent faire aussi l'objet d'évaluation d'impact afin d'évaluer leur efficacité et d'adapter le plan en conséquence.

Ainsi, les produits pour lesquels des risques importants ont été mis en évidence ou pressentis ou pour lesquels des données essentielles manquent, doivent faire l'objet de plans de gestion de risque préparés par l'industriel puis validés par les autorités de santé.

Parmi les actions d'identification et/ou de surveillance des risques à mettre en œuvre les études pharmaco-épidémiologiques, principalement études de cohorte, études cas-témoins, registres tiennent une grande place qu'elles soient de terrain ou réalisées à partir de bases de données. Ces recherches non interventionnelles, dites « études observationnelles», sont des études pour lesquelles la stratégie médicale destinée à une personne qui se prête à la recherche n'est pas fixée à l'avance par un protocole et relève de la pratique courante dans le cadre de laquelle le ou les médicament(s) sont prescrits de manière habituelle, conformément aux conditions fixées dans l'autorisation de mise sur le marché. Aucune procédure supplémentaire de diagnostic ou de surveillance ne doit être imposée aux patients.

Une contre-indication sous la rubrique Grossesse du Résumé des Caractéristiques du Produit (RCP) qui est une situation fréquente pour cette population, pourrait en théorie empêcher ces études observationnelles. La conduite de telles études n'est pas réglementairement permise, alors même qu'elles peuvent être requises, dans le cadre des plans de gestion de risque. Cette 
apparente contradiction devra faire l'objet de discussion préalable avec les autorités de santé, si l'on veut pouvoir mettre en place ces recherches.

\section{Ethique et essais cliniques chez la femme enceinte}

L'analyse des différents textes relatifs à l'expérimentation des médicaments chez la femme enceinte ou à l'utilisation de ces mêmes médicaments après leur mise sur le marché a permis de souligner des avancées mais aussi de relever certaines difficultés.

La loi relative à la politique de santé publique ${ }^{[13]}$ prévoit dans son article L1121-5 des dispositions pour cette population. Les femmes enceintes, les parturientes et les mères qui allaitent ne peuvent être sollicitées pour se prêter à des recherches biomédicales que dans les conditions suivantes :

- soit l'importance du bénéfice escompté pour elles-mêmes ou pour l'enfant est de nature à justifier le risque prévisible encouru;

- soit ces recherches se justifient au regard du bénéfice escompté pour d'autres femmes se trouvant dans la même situation ou pour leur enfant et à la condition que des recherches d'une efficacité comparable ne puissent être effectuées sur une autre catégorie de la population. Dans ce cas, les risques prévisibles et les contraintes que comporte la recherche doivent présenter un caractère minimal.

L'article 17 du protocole additionnel à la convention sur les droits de l'homme et la biomédecine relatif à la recherche biomédicale lors du Conseil de l'Europe du 25 janvier 2005 définit le risque minimal si, au regard de la nature et de la portée de l'intervention, on peut s'attendre à ce que la recherche entraîne, tout au plus, un impact négatif très faible et temporaire sur la santé de la personne concernée. De même, une contrainte minimale est caractérisée par le fait qu'on peut s'attendre à ce que les désagréments pouvant en résulter soient tout au plus temporaires et très légers pour la personne concernée.

Ainsi, il est éthiquement et réglementairement possible de réaliser des essais cliniques chez les femmes enceintes sous certaines conditions :

- une information préalable sur les risques potentiels et les bénéfices pour elles-mêmes, leur grossesse, leur fotus, leur progéniture future et pour leur fécondité, doit leur être donnée, orale et écrite, avec si possible un délai de réflexion;

- la recherche doit être pertinente pour les besoins de santé particuliers d'une femme enceinte ou de son fœus ou pour les besoins de santé des femmes enceintes en général;
- elle doit se fonder sur des expériences fiables réalisées sur des animaux évaluant notamment, les risques de tératogénicité et mutagénicité selon les lignes directrices internationales d'éthique pour la recherche biomédicale impliquant des sujets humains.

\section{Situations cliniques justifiant une surveillance des risques}

L'analyse du risque d'un médicament dans cette situation doit tenir compte de deux grands types de facteurs.

\section{1. Les données acquises sur le médicament}

- Classe thérapeutique et profil de risque toxicologique du médicament et des autres produits de la classe déjà sur le marché.

- Données pré cliniques disponibles pour le médicament considéré : comme précisé plus haut, les études expérimentales de tératogenèse ont une réelle valeur prédictive des effets tératogènes dans l'espèce humaine, alors que celles de toxicité du développement ne sont pas (ou peu) prédictives d'un risque toxique fœtal ou néonatal.

- Existence de données d'utilisation en thérapeutique humaine, incluant notamment les risques identifiés lors des essais thérapeutiques. Dans la mesure où les données chez la femme enceinte font généralement défaut, les données issues de la même classe thérapeutique et utilisés chez la femme enceinte seront également prises en compte.

\subsection{Son utilisation thérapeutique potentielle par des femmes en âge de procréer}

Différents paramètres doivent être analysés :

- l'épidémiologie de la maladie à traiter ;

- la probabilité d'exposition des femmes enceintes, dépendant notamment de la classe thérapeutique, de la place du «nouveau médicament» dans la prise en charge de la maladie, de l'existence d'alternatives thérapeutiques, en particulier de médicament de référence, ancien et «connu », pour lequel des données chez la femme enceinte sont disponibles...

- le type de pathologie maternelle prise en charge :

- pathologie aiguë, survenant en cours de grossesse, pour laquelle un traitement court sera prescrit ou parfois utilisé en automédication,

- pathologie chronique, connue avant le début de la grossesse, pouvant justifier d'une planification de la grossesse et d'éventuelles adaptations de traitements par exemple pour un médicament plus ancien et mieux connu... 
- pathologie gravidique...

- la possible exposition de la femme enceinte par le liquide séminal rend indispensable l'évaluation du risque mutagène si le médicament est utilisé par l'homme avant le début de la grossesse.

\section{Obtention des données humaines post-AMM}

Seules les données post-AMM portant sur un nombre suffisant de grossesses exposées permettent d'évaluer le risque d'un médicament chez la femme enceinte et le recueil des données d'exposition est donc essentiel. ${ }^{[14]}$

\subsection{Sources d'information}

- Notifications spontanées d'un effet indésirable par les professionnels de santé à une structure de Pharmacovigilance, laboratoire pharmaceutique ou Centre Régional de Pharmacovigilance (CRPV). Il s'agit de la déclaration d'un effet indésirable chez la mère ou chez le nouveau-né ayant été exposé in utero. Les notifications spontanées d'exposition, et non d'un effet indésirable, sont particulières à la grossesse (et à un moindre degré à la pédiatrie). Elles sont souvent motivées par une demande d'évaluation du risque potentiel, en particulier du risque malformatif. Les données sont parfois incomplètes et l'analyse du lien de causalité doit être prudente. Si ces signalements isolés sont insuffisants dans la plupart des cas pour mesurer le risque, ils restent indispensables pour générer un signal. Ainsi la répétition de signalements identiques pour une molécule ou une classe thérapeutique donnée peut suffire à identifier un risque embryo-fœtal et prendre les mesures appropriées (par exemple anti-inflammatoires non stéroïdiens [AINS] et toxicité rénale et cardio-pulmonaire, inhibiteurs de l'enzyme de conversion [IEC] ou sartans et toxicité rénale). De nombreux exemples illustrent les biais liés à l'analyse rétrospective de ces données. Ainsi un risque élevé de malformations notamment cardiaques compliquant la prise maternelle de lithium pendant la grossesse, avait été suspecté par l'analyse d'un registre constitué à partir de notifications spontanées. La conduite d'une étude prospective a permis de vérifier que le risque initial avait été surestimé. ${ }^{[15,16]}$ Des structures spécialisées actuellement présentes dans de nombreux pays, renseignent les professionnels de santé et les patientes sur les risques d'une exposition pendant la grossesse et assurent le suivi prospectif des demandes d'information. Ils recueillent des informations sur le déroulement et l'issue de la grossesse, l'examen du nouveau-né avec parfois un suivi à distance de l'enfant. ${ }^{[17]}$ Les études réalisées sont dans la majorité des cas collaboratives et comportent de plus en plus fréquemment un groupe de comparaison constitué par des patientes exposées à des substances non tératogènes ou à d'autres médicaments ayant la même indication thérapeutique. L'effectif de patientes exposées est parfois insuffisant pour conclure définitivement à une absence de risque, néanmoins il s'agit de premiers résultats intéressants lorsque aucune autre donnée clinique n'est disponible. L'absence d'augmentation du risque d'anomalie ou de complication par rapport au taux attendu dans une population de référence ou dans le groupe de comparaison ne permet pas de conclure à l'innocuité du médicament concerné mais élimine un risque important. Ce type d'étude permet par ailleurs d'analyser des paramètres rarement pris en compte, comme le risque de fausse couche spontanée ou le taux d'interruption de grossesse.

- Surveillance active programmée.

La surveillance active permet de conduire des études épidémiologiques, comme des études cas - témoins ou étude de cohortes. Elle s'appuie sur différents outils :

- les sites sentinelles, où peuvent être réalisées des analyses systématiques et exhaustives des dossiers et/ou des interrogatoires de tous les patients. Dans le cadre de la grossesse, ces sites sentinelles sont les maternités où les grossesses exposées à un médicament donné peuvent être suivies de manière prospective ;

- les registres, dans le cadre de la grossesse peuvent concerner les malformations, l'exposition aux médicaments ou une une pathologie spécifique. Plusieurs registres de malformations existent en France : celui du Centre-Est a été à l'origine de la démonstration de l'implication de l'acide valprö̈que dans la survenue de spina bifida. ${ }^{[18]}$

Au niveau international, les différents registres créés à partir des années 60 sont regroupés en organisations internationales telles que EUROCAT et l'International Clearinghouse (ICBDMS). Dans ces deux systèmes, l'incidence de quelques malformations sélectionnées est calculée régulièrement, et des méthodes statistiques simples sont appliquées pour déterminer si des changements significatifs ont eu lieu. Ainsi peut être détecté le changement d'incidence d'une malformation rare. Mais cela présuppose que le médicament tératogène éventuel a un effet aussi puissant que la thalidomide ou l'isotretinoïne.

La surveillance en routine de l'effet tératogène des médicaments est assurée par une méthode originale d'analyse des données recueillies par ces registres (projet MADRE MAlformation DRug Exposure Surveillance de l'ICBDMS).

Cependant toute association trouvée significative entre un médicament et une malformation doit être interprétée avec 
précaution et testée sur d'autres séries de cas puis servir d'hypothèse pour une étude épidémiologique $a d$ hoc. Ce n'est qu'après confrontation aux études expérimentales que l'effet tératogène pourra être confirmé.

- Les études de recherche clinique chez la femme enceinte sont indispensables pour étudier les modifications pharmacologiques liées à la grossesse et leurs implications en thérapeutique. Elles concernent les traitements des pathologies maternelles chroniques, maintenus lors de la grossesse. Il peut s'agir d'études pharmacocinétiques afin dévaluer les modifications du devenir maternel d'un médicament ou de son transfert placentaire et de sa distribution dans l'unité fœtoplacentaire. ${ }^{[19]}$

Il peut s'agir d'un réel essai thérapeutique, pour la prise en charge d'une pathologie gravidique. Dans ces situations, la complexité du protocole est liée à la difficulté de sélectionner les paramètres de bénéfice et de risque fœtal. Enfin les études pharmacogénétiques doivent être réalisées pour identifier les marqueurs génétiques de risque en particulier de malformation fœtale. Dans ce domaine, seules de larges études de population pourront rassembler un nombre suffisant de patients (couples parents - enfant) nécessaires à une analyse pharmacogénétique rétrospective.

\subsection{Les acteurs impliqués au niveau national}

Au plan national, de nombreux acteurs tant privés que publiques sont impliqués à des degrés divers dans le recueil, l'évaluation des médicaments chez la femme enceinte puis l'information aux professionnels de santé ainsi qu'aux patientes.

\subsubsection{Structures de pharmacovigilance}

a) Le réseau national de Pharmacovigilance

Les centres régionaux de pharmacovigilance sont au nombre de 31. Leur mission est de recueillir, analyser et assurer le suivi des effets indésirables notifiés par les praticiens de la région qu'ils couvrent.

Conformément au décret du 26 mai 2005 portant sur les bonnes pratiques de pharmacovigilance, les centres doivent aussi enregistrer toute information relative à une utilisation de médicament lors de la grossesse et assurer le suivi de ces demandes afin de documenter l'issue de la grossesse. Un nombre grandissant de centres de pharmacovigilance enregistre ces informations sur une banque de donnée informatisée spécifique et commune, ces données pouvant être ultérieurement utilisées dans le cadre d'études collaboratives [ $c f .5 .1]$ ou pour fournir des informations rapides à l'Afssaps (Agence Française de Sécurité Sanitaire des produits de Santé) en cas d'alerte. Certains d'entre eux sont aussi membres d'un réseau européen de services d'informations sur les agents tératogènes.

Certains centres de pharmacovigilance qui ont un intérêt et une reconnaissance dans le domaine "grossesse et pédiatrie » pourraient bénéficier d'un affichage clair de leurs compétences dans ce domaine. La possibilité et même l'intérêt d'un tel affichage ont été débattus mais n'ont pas fait l'objet d'un consensus. Ces centres ont développé une application informatique (TERAPPEL) permettant la saisie et le suivi de ces observations de grossesse.

b) Centres de renseignements «grossesse »

- Le Centre de Renseignements sur les Agents Tératogènes (CRAT) informe sur les risques de divers agents en cours de grossesse (médicaments, radiations, virus, expositions professionnelles et environnementales...).

- Information sur les médicaments administrés pendant la grossesse et chez l'enfant-IMAGE- Il s'agit d'une structure de renseignements sur « Médicaments et Grossesse » dont la vocation principale est de développer une activité de recherche clinique. Une consultation de femmes enceintes est conduite à la demande dans la maternité.

\section{c) Centres anti-poison}

Les centres anti-poison (CAP) sont probablement moins fréquemment sollicités que les centres de pharmacovigilance. Ils disposent cependant d'informations utiles à l'évaluation, notamment par le suivi des demandes concernant une ingestion médicamenteuse à visée suicidaire au cours de la grossesse. Par ailleurs, ils ont l'expérience de la gestion et du suivi des demandes provenant du grand public.

d) Départements de Pharmacovigilance des laboratoires pharmaceutiques

Ces départements ont pour mission de surveiller les médicaments mis sur le marché par la firme à laquelle ils appartiennent. Ils peuvent renseigner les praticiens qui les contactent et assurent le suivi des observations Les firmes multinationales ont ainsi une large base de données. En général, les firmes ne colligent que les observations qui leur sont notifiées, mais elles peuvent aussi mettre en place des registres dans un ou plusieurs pays.

Les données nationales de pharmacovigilance des firmes sont transmises à l'Afssaps, au même titre que les données des centres de pharmacovigilance.

\section{e) Les registres de malformation}

Il en existe 4 en France : Paris, Rhône-Alpes, Auvergne et Alsace. Ils enregistrent tous les cas de malformations d'une région donnée, à la naissance ou chez des fœtus après interruption 
de grossesse. Le recueil des expositions médicamenteuses est fait de manière rétrospective, au moment de l'enregistrement du cas, et il est limité au premier trimestre de la grossesse. L'exploitation des données est faite par chaque registre d'une part, et au centre international d'étude des malformations de Rome, auquel les registres français contribuent.

f) Les réseaux de maternité/néonatalogie

Un exemple est le réseau AUDIPOG. Il est constitué, depuis 1994, d'un ensemble de maternités volontaires publiques et privées, provenant de toutes les régions de France, utilisant ou non un dossier obstétrical AUDIPOG, informatisé ou non.

\subsubsection{Objectifs et activités}

Les objectifs du réseau, définis lors de sa création, étaient les suivants :

- surveiller, année après année, les indicateurs de santé périnatale (indicateurs de recrutement, de pratiques et de résultats), par une mise en commun des données issues des fichiers des maternités participant au Réseau ;

- fournir aux maternités un outil d'évaluation de leurs pratiques et de leurs résultats, en comparant les résultats des analyses pratiquées sur leurs propres fichiers à ceux du Réseau Sentinelle;

- mettre en place des études multicentriques, à la demande d'un ou plusieurs membres du Réseau.

Ces entités apparaissent donc très différents sur le plan des objectifs et des activités. Ceux-ci ont été analysés, permettant d'identifier les paramètres suivants :

- type de questions posées et de données recueillies :

- expositions/suivi prospectif concernant l'issue de grossesse et le statut du nouveau-né,

- notifications d'effets indésirables quel qu'en soit le type : malformation, effet toxique, fotal, néonatal...

- type d'appels : local, régional, national...

- informatisation,

- activité de conseil / information par téléphone, par courrier,

- consultations, participation aux staffs médicaux,

- mise en commun des données, transmission...

Il apparaît donc que toutes les structures impliquées colligent des données obtenues par notification spontanée et/ou sollicitée. Elles assurent aussi une mission d'information sur « Médicaments et Grossesse » auprès des médecins et/ou directement du public. L'activité «Grossesse » peut être prédominante voire exclusive, ou s'inscrire dans le cadre d'une activité «non spécialisée » de pharmacovigilance. Tous les centres assurent de manière aussi exhaustive que possible, le suivi des observations pour obtenir l'issue de la grossesse. Néanmoins, l'absence de contact direct avec les médecins et ou les patients peut, dans certaines structures, gêner l'obtention des données de suivi, et rendre difficile leur validation.

\section{Recommandations}

\section{1. Pour répondre à un plan de gestion du risque}

Un plan de gestion de risque ayant pour but une meilleure surveillance du médicament après sa mise sur le marché, est défini comme un processus global et permanent de gestion, si possible de minimisation des risques tout au long de la vie des médicaments afin d'optimiser leur rapport bénéfice/risque.

Il doit répondre de manière optimale à des situations très variées, qui dépendent du médicament, de sa place dans la thérapeutique en dehors de la grossesse, de son profil toxicologique etc. Il n'est donc pas possible de prévoir un plan de gestion de risques «standard» adapté à tous les médicaments/toutes les situations à risque. Il est par contre possible de dresser les objectifs et les grandes lignes d'organisation permettant de répondre à différentes situations.

Il faut tenir compte d'arguments pharmacologiques, des données expérimentales et de risques toxiques mis en évidence dans d'autres populations (adulte, enfant...).

Il peut donc être planifié a minima (simple recueil de données d'exposition par les systèmes de pharmacovigilance en place) ou au contraire exiger un suivi optimisé voire exhaustif. Dans ces deux cas extrêmes, la réponse est une optimisation des performances des systèmes existants, en terme de rapidité de réponse et de transparence.

Comme souligné plus haut, les structures impliquées sont multiples et les données sont éparpillées, non ou tardivement accessibles.

Une mutualisation et une coordination des systèmes de surveillance existants doivent permettre de satisfaire des objectifs simples :

- mieux surveiller et analyser les données, et notamment analyser systématiquement les premières expositions. Des méthodes statistiques de détection de signal, en cours d'évaluation, peuvent être utilisées pour faciliter l'évaluation des notifications spontanées ; ${ }^{[20,21]}$

- répondre rapidement à une alerte (situation rare en pratique...);

- anticiper ou générer l'alerte (probabilité de risque) et la non alerte (probabilité de non risque); 
- diffuser des informations validées et harmonisées aux spécialistes.

Ceci suppose une organisation en réseau réactive, non centralisée, associant la firme et une structure de pharmacovigilance.

\subsection{Organiser un réseau Mère-Enfant en France}

Idéalement, une organisation qui permettrait le recueil de toutes les expositions médicamenteuses (et toxiques) des femmes à chaque visite prénatale, s'appuyant sur le carnet de maternité aurait l'avantage d'être prospectif, et donc d'éviter tous les biais liés au caractère rétrospectif des données. Une telle organisation a été développée en Suède.

Compte tenu de la situation française, la mise en place d'un réseau Mère-Enfant nécessite la création d'un comité de coordination qui a les objectifs suivants :

- fédérer les différents partenaires en y intégrant les réseaux médicaux et les structures implantées dans les hôpitaux et les cliniques ouverts aux spécialistes impliqués gynécologues obstétriciens, pharmacologues, industriels... pour définir les objectifs, les modalités de mise en place;

- préciser les relations du réseau avec les agences nationales et européennes du médicament, les liens avec les départements de pharmacovigilance des industries pharmaceutiques et l'Assurance Maladie ;

- harmoniser les données recueillies initialement concernant la mère, la grossesse, le fœus ;

- définir les modalités du suivi de la grossesse, les paramètres recueillis (en particuliers néonatals), la nécessité d'un suivi prolongé...

- établir une charte informatique permettant d'uniformiser les données recueillies, de mettre en place un contrôle qualité, de préciser les délais de saisie ;

- établir la charte de fonctionnement du réseau et en particulier de définir les données échangeables, particulierement l'accessibilité des données, la réalisation d'études ad hoc conditions d'accessibilité, transmission à l'Afssaps.

\subsubsection{Sollicitation du réseau dans le cadre d'un plan de gestion de risque}

La figure 1 illustre le circuit de sollicitation du réseau national Mère-Enfant lors de la mise en place d'un plan de gestion de risque.

- Le réseau peut être activé par la détection précoce d'un signal sur un médicament. L'analyse des tout premiers cas est confié à un comité scientifique prenant en charge l'analyse de toutes les notifications (positives et négatives) signalées dans le réseau et correspondant à cette alerte.

- Le réseau peut aussi être sollicité par un de ses membres pour conduire une étude utilisant la base de données du réseau. Les objectifs de l'étude, les modalités de participation de chacun sont proposés par le comité scientifique de l'étude, coordonné par l'investigateur principal et accepté par le comité de coordination du réseau.

\subsection{Le futur du réseau Mère-Enfant}

Une fois organisé, le réseau Mère-Enfant pourra répondre à deux besoins majeurs.

\subsubsection{L'aide à la mise en place et à la réalisation d'études collaboratives en pharmacovigilance}

Les partenaires du réseau issus d'horizons très variés pourront :

- soulever les questions pertinentes,

- fournir une aide méthodologique (type d'étude adaptée, choix d'une population témoin...),

- organiser, standardiser et conduire des protocoles de recherche clinique : études cinétiques chez la femme enceinte ou qui allaite, le nouveau-né..., banques de prélèvements...

\subsubsection{Diffusion de l'information aux professionnels de santé et aux patientes}

A l'heure actuelle, lorsque les données scientifiques sont disponibles, l'information transmise par les différents acteurs peut paraître confus, hésitant et aboutir à des décisions individuelles inadaptées. L'aide à la gestion du risque individuel, qu'il s'agisse du risque d'une exposition fortuite, de l'évaluation des risques d'une prescription ou de modification d'un traitement chronique en vue d'une grossesse, doit donc être un objectif prioritaire du réseau. L'enjeu de l'information est essentiel et celle-ci doit être harmonisée et fournie par des médecins formés.

\section{Conclusion}

La mise en place des plans de gestion du risque est devenue une obligation réglementaire depuis novembre 2005. Leur application à la femme enceinte est particulièrement importante en l'absence de toute donnée humaine disponible. Mais les situations cliniques variées ne rendent pas possibles la mise en œuvre 


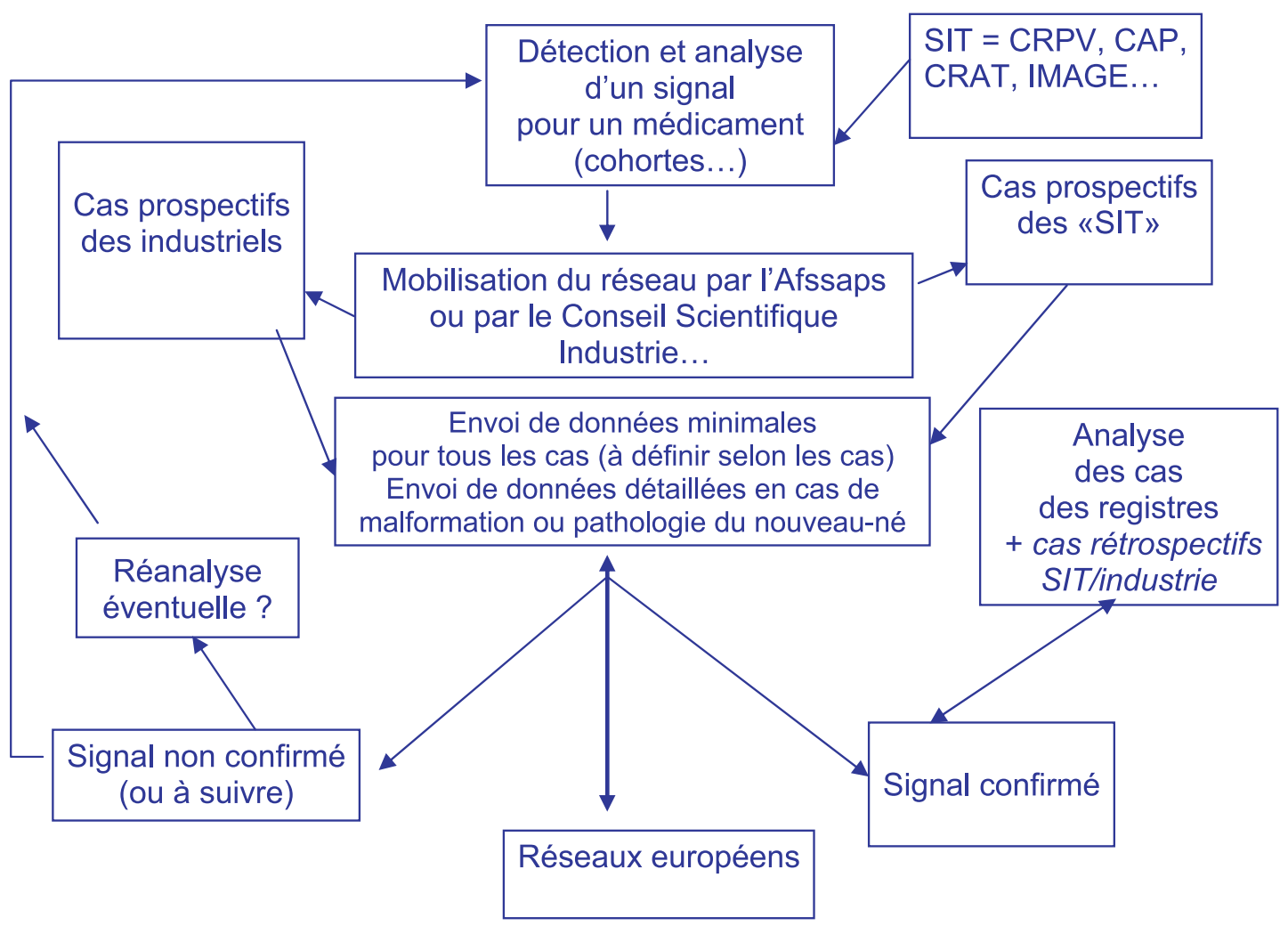

Fig. 1. Ce que l'on peut faire rapidement : officialiser un réseau Mère-Enfant. SIT : Services d'informations sur les tératogènes; CRPV : Centre Régional de Pharmacovigilance; CAP : Centre Anti-Poison; CRAT : Centre de Renseignements sur les Agents Tératogènes; Afssaps : Agence Française de Sécurité Sanitaire des Produits de Santé.

de plans standardisés. Néanmoins il est pratiquement toujours nécessaire d'organiser un suivi des expositions voire conduire des études épidémiologiques en collaboration avec les structures spécialisées. Au niveau national de nombreuses structures sont impliquées pour le suivi des grossesses et des nouveaux-nés. Leur organisation en réseau permettrait à ces structures de disposer de l'ensemble des informations disponibles sous une forme standardisée et ainsi d'être plus réactives.

\section{Participants}

E. Autret-Leca (Hôpital Bretonneau, Tours), L. Becquemont (CHU Bicêtre, Le Kremlin-Bicêtre), M.-J. Boutroy, (Maternité Régionale Universitaire, Nancy), P. Carlier (Hôpital Fernand Widal, Paris), A. Castot (Afssaps) C. Cornu (CIC Hôpital Louis Pradel, Bron), C. Damase-Michel (Faculté de Médecine, Toulouse), J.-P. Demarez (Pierre Fabre Médicaments), E. Dohin (Merck Sharpe \& Dohme Chibret), M. Gersberg (Sanofi-Aventis) C. Kreft-Jais (Afssaps) H. Le Louet (Hôpital Henri Mondor, Créteil) F. Meillier (LEEM), J.-L. Parier (Boehringer Ingelheim France), G. Pons (Hôpital Saint Vincent de Paul, Paris), D. Subtil
(Hôpital Jeanne de Flandre, Lille), T. Vial (Hôpital Edouard Herriot, Lyon). Nous remercions les membres de la table ronde qui ont participé à la rédaction de cette synthèse.

\section{Références}

1. Beyens MN, Guy C, Ratrema M, et al. Prescription médicamenteuse pendant la grossesse en France : étude HIMAGE. Thérapie 2003; 58: 505-11

2. Felix RJ, Jones KL, Johnson KA, et al. Post marketing surveillance for drug safety in pregnancy: the organization of teratology information services project. Birth Defects Research 2004; 70: 944-7

3. Lacroix I, Damase-Michel C, Lapeyre-Mestre M, et al.. Prescription of drugs during pregnancy in France. Lancet 2000; 356: 1735-6

4. Brocklebank JC, Ray WA, Federspiel CF, et al. Drug prescribing during pregnancy. A controlled study of Tennessee Medicaid recipients. Am J Obstet Gynecol 1978; 132: 235-44

5. Piper JM, Baum C, Kennedy DL. Pescription drug use before and during pregnancy in a Medicaid population. Am J Obstet Gynecol 1987; 157: 148-56

6. Rubin JD, Ferencz C, Loffredo C. Use of prescription and non-prescription drugs in pregnancy: the Baltimore-Washington Infant Study Group. J Clin Epidemiol 1993; 46: 581-9

7. Boethius G. Recording of drug prescriptions in the county of Jamtland, Sweden. II. Drug exposure of pregnant women in relation to course and outcome of pregnancy. Eur J Clin Pharmacol 1977; 12: 37-43

8. Guittin P, Soubrie C, Autret E, et al. Toxicologie de la reproduction : valeurs prédictives des modèles expérimentaux. Thérapie 1998; 53: 355-63 
9. Elefant E, Sainte-Croix A. Evaluation du risque médicamenteux chez la femme enceinte : méthodologie d'evaluation et gestion du risque. Thérapie 1997; 52: $307-11$

10. Chauvenet M, Rimailho A, Hoog-Labouret N. Methodology for the evaluation of drugs in pregnant women. Thérapie 2003; 58: 247-58

11. Conférence Internationale d'Harmonisation, ICH. http://www.ich.org

12. Guideline on risk management systems for medicinal products for human use, EMEA/CHMP/96268/2005 http: //www . emea . eu . int

13. Loi 2004-806 du 9 août 2004 du Code de Santé Publique http://www.legifrance.gouv

14. Note for guidance on the exposure to medicinal products during pregnancy: need for post-authorisation data, EMEA/CHMP/1889/04 http://www. emea.eu.int

15. Weinstein RM, Goldfield M. Cardiovascular malformations with lithium use during pregnancy. Am J Psychiatry 1975, 132: 529-31

16. Jacobson SJ, Jones K, Johnson K, et al. Prospective multicenter study of pregnancy after lithium exposure during first trimester. Lancet 1992; 339: 530-3

17. Clementi M, Di Gianantonio E, Ornoy A. Teratology information services in Europe and their contribution to the prevention of congenital anomalies. Community Genet 2002; 5: 8-12
18. Robert-Gnansia E. Méthodes d'évaluation des risques liés aux prises médicamenteuses pendant la grossesse. Avantages et limites de l'épidémiologie, interprétation des résultats. Thérapie 2002; 57: 175-80

19. Koren G. Clinical trials during pregnancy: Challenges and solutions. In Paediatric Clinical Pharmacology, p 359-365, 2005 edited by E. JacqzAigrain, I Choonara, Fontis Media Editors

20. Bates DW, Pappius EM, Kuperman GJ, et al. Medinfo. Measuring and improving quality using information systems 1998; 9: 814-8

21. Evans SJ, Waller PC, Davis S. Use of proportional reporting ratios (PRRs) for signal generation from spontaneous adverse drug reaction reports. Pharmacoepidemiol Drug Saf 2001; 10: 483-6

Correspondance et offprints : Evelyne Jacqz Aigrain, Service de Pharmacologie Pédiatrique et Pharmacogénétique, Hôpital Robert Devré, 48 boulevard Sérurier, 75019 Paris, France.

E-mail : evelyne.jacqz-aigrain@rdb.aphp.fr 